International Journal of Pure and Applied Mathematics

Volume 104 No. 4 2015, 523-532

ISSN: 1311-8080 (printed version); ISSN: 1314-3395 (on-line version)

url: http://www.ijpam.eu

doi: http://dx.doi.org/10.12732/ijpam.v104i4.4

ijpam.eu

\title{
NEW TRAVELLING WAVE SOLUTIONS FOR STOCHASTIC CUBIC NON LINEAR KLEIN-GORDON EQUATION
}

\author{
Hossam A. Ghany ${ }^{1,2} \S$, M.R. Al Harthi ${ }^{2}$, M. Al Qurashi² \\ ${ }^{1}$ Department of Mathematics \\ Helwan University \\ Cairo, 11282, EGYPT \\ ${ }^{2}$ Department of Mathematics \\ Taif University \\ Taif, 888, SAUDI ARABIA
}

\begin{abstract}
This paper is devoted to give white noise functional solutions for stochastic cubic nonlinear Klein-Gordon equation. Abundant exact travelling wave solutions for Wick-type generalized stochastic Klein-Gordon equation are obtained. By using white noise analysis, Hermite transform, modified Riccati equation and modified tanh-coth method many exact travelling wave solutions are given. Detailed computations and implemented examples for the investigated model are explicitly provided.
\end{abstract}

AMS Subject Classification: 60H30, 60H15, 35R60

Key Words: white noise, stochastic, wick product, Klein-Gordon equations

\section{Introduction}

The main aim of this paper is to investigate the generalized variable coefficient Klein-Gordon (KG) equation:

$$
u_{t t}-\omega(t) u_{x x}+\phi(t) u-\psi(t) u^{3}-\theta(t) u^{5}=0, \quad(x, t) \in \mathbb{R} \times \mathbb{R}_{+}
$$

where $u$ is a stochastic process on $\mathbb{R} \times \mathbb{R}_{+}$and $\phi(t), \psi(t)$ and $\theta(t)$ are bounded

Received: May 17, 2015

(c) 2015 Academic Publications, Ltd.

$\S$ Correspondence author url: www.acadpubl.eu 
measurable or integrable functions on $\mathbb{R}_{+}$. Equation (1.1) plays a significant role in many scientific applications such as solid state physics, nonlinear optics, chemical kinetics, the quantum field theory etc. The KG equations[1-2] are universal models(normal forms) for the propagation of long, dispersive, weakly nonlinear waves that travel predominantly in the $x$ direction, with weak transverse effects. The notion of well-posed-ness will be the usual one in the context of nonlinear dispersive equations, that is, it includes existence, uniqueness, persistence property, and continuous dependence upon the data. Recently, many researchers pay more attention to study of random waves, which are important subjects of stochastic partial differential equation (SPDE). Wadati[3] first answered the interesting question, How does external noise affect the motion of solitons? and studied the diffusion of soliton of the $\mathrm{KdV}$ equation under Gaussian noise, which satisfies a diffusion equation in transformed coordinates. Wadati and Akutsu also studied the behaviors of solitons under the Gaussian white noise of the stochastic KdV equations with and without damping[4]. Wadati[3] first answered the interesting question, "How does external noise affect the motion of solitons?" and studied the diffusion of soliton of the KdV equation under Gaussian noise, which satisfies a diffusion equation in transformed coordinates. The stochastic PDEs was discussed by many authors, e.g., Holden et al.[5], de Bouard and Debussche [6,7] and Debussche and Printems [8,9]. On the basis of white noise functional analysis [5], Ghany et al. [10-13] studied more intensely the white noise functional solutions for some nonlinear stochastic PDEs. This paper is mainly concerned to investigate the white noise functional solutions for the generalized Wick-type stochastic Klein-Gordon (KG) equation:

$$
U_{t t}-\Omega(t) \diamond U_{x x}+\Phi(t) \diamond U-\Psi(t) \diamond U^{\diamond 3}+\Theta(t) \diamond U^{\diamond 5}=0 .
$$

where " $\diamond$ " is the Wick product on the Kondratiev distribution space $(\mathcal{S})_{-1}$ and $\Phi(t), \Psi(t)$ and $\Theta(t)$ are $(\mathcal{S})_{-1}$-valuedfunctions [5]. It is well known that the solitons are stable against mutual collisions and behave like particles. In this sense, it is very important to study the nonlinear equations in random environment. However, variable coefficients nonlinear equations, as well as constant coefficients equations, cannot describe the realistic physical phenomena exactly. The rest of this paper is organized as follows: In Section 2, we recall the definition and some properties of white noise analysis. In Section 3, we apply some method to explore exact travelling wave solutions for Eq.(1.1). In Section 4, we use the Hermite transform and [5, Theorem 4.1.1] to obtain white noise functional solutions for Eq.(1.2). In Section 5, we give illustrative examples for the investigated model. The last section is devoted to summary and discussion. 


\section{Exact Travelling Wave Solutions}

In this section, we will give exact solutions of Eq.(1.1). Taking the Hermite transform of Eq.(1.2), we get:

$$
\begin{aligned}
\widetilde{U}_{t t}(t, x, z)-\widetilde{\Omega}(t, z) \cdot \widetilde{U}_{x x}(t, x, z)+\widetilde{\Phi}(t, z) \cdot \widetilde{U}(t, x, z) \\
-\widetilde{\Psi}(t, z) \cdot \widetilde{U}^{3}(t, x, z)+\widetilde{\Theta}(t) \cdot \widetilde{U}^{5}(t, x, z)=0 .
\end{aligned}
$$

where $z=\left(z_{1}, z_{2}, \ldots\right) \in \mathbb{C}^{\mathbb{N}}$ is a parameter. To look for the travelling wave solution of Eq.(2.1),we make the transformations $u(t, x, z):=\widetilde{U}(t, x, z)=$ $\varphi(\xi(t, x, z))$ with

$$
\xi(t, x, z):=k x+s \int_{0}^{t} l(\tau, z) d \tau+c
$$

where $k_{1}, s, c$ are arbitrary constants which satisfy $k_{1} s \neq 0, l(\tau, z)$ is a non zero functions of indicated variables to be determined. So, Eq.(2.1) can be changing into the form:

$$
\left(s l-k^{2} \omega\right) u^{\prime \prime}(t, x, z)+\phi u(t, x, z)-\psi u^{3}(t, x, z)+\theta u^{5}(t, x, z)=0
$$

The solution can be proposed by the tanh method as a finite power series in $\mathrm{Y}$ in the form:

$$
u(\mu \zeta)=S(Y)=\sum_{k=0}^{M} A_{k} Y^{k},
$$

limiting them to solitary and shock wave profiles. However, the extended tanh method admits the use of the finite expansion

$$
u(\mu \zeta)=S(Y)=\sum_{k=0}^{M} A_{k} Y^{k}+\sum_{k=1}^{M} B_{k} Y^{-k},
$$

where $\mathrm{M}$ is a positive integer, in most cases, that will be determined by using homogenous balance principle. Expansion (3.2) reduces to the standard tanh method [1]. Applying, the homogenous balance principle, we obtain $\mathrm{M}=0.5 \notin$ $\mathbb{N}$. Suppose that

$$
u(\xi)=[\chi(\xi)]^{\frac{1}{2}}
$$

Substituting from (2.5) into (2.2) we get the following new ODE:

$$
\frac{\left(s l-k^{2} \omega\right)}{4}\left[2 \chi \chi^{\prime \prime}(\xi)-\chi^{\prime 2}\right]+\phi \chi^{2}(\xi)-\psi \chi^{3}(\xi)+\theta \chi^{4}(\xi)=0
$$


Applying homogenous balance, the solution can be written in the form:

$$
\chi(\xi)=A_{0}+A_{1} \Lambda(\xi)+\frac{B_{1}}{\Lambda(\xi)}
$$

Where $\chi(\xi)$ satisfies the Riccati equation

$$
\Lambda^{\prime}=c_{0}+c_{1} \Lambda+c_{2} \Lambda^{2}
$$

and $c_{0}, c_{1}, c_{2}$ are constant to be prescribed later. By virtue of (2.7) and (2.8) with observation of the linear independence of $\Lambda^{n}(n=-4,-3, \ldots, 4)$ and using Mathematica Eqn.(2.6) implies the following nonlinear algebraic system of equations:

$$
\left\{\begin{array}{l}
\frac{\left(s l-k^{2} \omega\right)}{4}\left[2\left(A_{0} \alpha_{2,0}+A_{1} \alpha_{2,-1}+B_{1} \alpha_{2,1}\right)-\left(\alpha_{1,0}^{2}+2 \alpha_{1,1} \alpha_{1,-1}+2 \alpha_{1,2} \alpha_{1,-2}\right)\right] \\
+{ }_{2} \lambda_{0} \Phi-{ }_{3} \lambda_{0} \Psi+{ }_{4} \lambda_{0} \Theta=0 \\
\frac{\left(s l-k^{2} \omega\right)}{4}\left[2\left(A_{0} \alpha_{2,1}+A_{1} \alpha_{2,0}+B_{1} \alpha_{2,2}\right)-\left(2 \alpha_{1,1} \alpha_{1,0}+2 \alpha_{1,2} \alpha_{1,-1}\right)\right] \\
+{ }_{2} \lambda_{1} \Phi-{ }_{3} \lambda_{1} \Psi+{ }_{4} \lambda_{1} \Theta=0 \\
\frac{\left(s l-k^{2} \omega\right)}{4}\left[2\left(A_{0} \alpha_{2,2}+A_{1} \alpha_{2,1}\right)-\left(2 \alpha_{1,0} \alpha_{1,2}+\alpha_{1,1}^{2}\right)\right] \\
+{ }_{2} \lambda_{2} \Phi-{ }_{3} \lambda_{2} \Psi+{ }_{4} \lambda_{2} \Theta=0 \\
\frac{\left(s l-k^{2} \omega\right)}{4}\left[2\left(A_{1} \alpha_{2,2}\right)-\left(2 \alpha_{1,1} \alpha_{1,2}\right)\right]-{ }_{3} \lambda_{3} \Psi+{ }_{4} \lambda_{3} \Theta=0 \\
\frac{\left(s l-k^{2} \omega\right)}{4}\left[2\left(A_{1} \alpha_{2,3}\right)-\left(\alpha_{1,2}^{2}\right)\right]+{ }_{4} \lambda_{4} \Theta=0 \\
\frac{\left(s l-k^{2} \omega\right)}{4}\left[2\left(A_{0} \alpha_{2,-1}+A_{1} \alpha_{2,-2}+B_{1} \alpha_{2,0}\right)-\left(2 \alpha_{1,0} \alpha_{1,-1}+2 \alpha_{1,1} \alpha_{1,-2}\right)\right] \\
+{ }_{2} \lambda_{-1} \Phi-{ }_{3} \lambda_{-1} \Psi+{ }_{4} \lambda_{-1} \Theta=0 \\
\frac{\left(s l-k^{2} \omega\right)}{4}\left[2\left(A_{0} \alpha_{2,-2}+A_{1} \alpha_{2,-3}+B_{1} \alpha_{2,-1}\right)-\left(2 \alpha_{1,0} \alpha_{1,-2}+\alpha_{1,-1}^{2}\right)\right] \\
+{ }_{2} \lambda_{-2} \Phi-{ }_{3} \lambda_{-2} \Psi+{ }_{4} \lambda_{-2} \Theta=0 \\
\frac{\left(s l-k^{2} \omega\right)}{4}\left[2\left(A_{0} \alpha_{2,-3}+B_{1} \alpha_{2,-2}\right)-\left(2 \alpha_{1,-1} \alpha_{1,-2}\right)\right]-{ }_{3} \lambda_{-3} \Psi+{ }_{4} \lambda_{-3} \Theta=0 \\
\frac{\left(s l-k^{2} \omega\right)}{4}\left[2\left(B_{1} \alpha_{2,-3}\right)-\left(\alpha_{1,-2}^{2}\right)\right]+{ }_{4} \lambda_{-4} \Theta=0
\end{array}\right.
$$


where

$$
\left\{\begin{array}{l}
\alpha_{1,0}=A_{1} c_{0}-B_{1} c_{2}, \quad \alpha_{1,1}=A_{1} c_{1}, \quad \alpha_{1,2}=A_{1} c_{2}, \\
\alpha_{1,-1}=-B_{1} c_{1}, \quad \alpha_{1,-2}=-B_{1} c_{0}, \\
\alpha_{2,0}=\alpha_{1,1} c_{0}-\alpha_{1,-1} c_{2}, \quad \alpha_{2,1}=\alpha_{1,1} c_{1}+2 \alpha_{1,2} c_{0}, \\
\alpha_{2,2}=\alpha_{1,1} c_{2}+2 \alpha_{1,2} c_{1}, \\
\alpha_{2,3}=2 \alpha_{1,2} c_{2}, \alpha_{2,-1}=-\left(\alpha_{1,-1} c_{1}+2 \alpha_{1,-2} c_{2}\right) \\
\alpha_{2,-2}=-\left(\alpha_{1,-1} c_{0}+2 \alpha_{1,-2} c_{1}\right), \quad \alpha_{2,-3}=-\left(2 \alpha_{1,-2} c_{0}\right) \\
{ }_{2} \lambda_{0}=A_{0}^{2}+2 A_{1} B_{1}, \quad{ }_{2} \lambda_{1}=2 A_{0} A_{1}, \\
{ }_{2} \lambda_{2}=A_{1}^{2}, \quad{ }_{2} \lambda_{-1}=2 A_{0} B_{1}, \quad{ }_{2} \lambda_{-2}=B_{1}^{2}, \\
{ }_{3} \lambda_{0}=A_{02} \lambda_{0}+A_{12} \lambda_{-1}+B_{12} \lambda_{1}, \quad{ }_{3} \lambda_{1}=A_{02} \lambda_{1}+A_{12} \lambda_{0}+B_{12} \lambda_{2}, \\
{ }_{3} \lambda_{2}=A_{02} \lambda_{2}+A_{12} \lambda_{1}, \\
{ }_{3} \lambda_{3}=A_{12} \lambda_{2}, \quad{ }_{3} \lambda_{-1}=A_{02} \lambda_{-1}+A_{12} \lambda_{-2}+B_{12} \lambda_{0}, \\
{ }_{3} \lambda_{-2}=A_{02} \lambda_{-2}+A_{12} \lambda_{2}, \quad{ }_{3} \lambda_{-3}=B_{12} \lambda_{-2} \\
{ }_{4} \lambda_{0}=A_{03} \lambda_{0}+A_{13} \lambda_{-1}+B_{13} \lambda_{1}, \quad{ }_{4} \lambda_{1}=A_{03} \lambda_{1}+A_{13} \lambda_{0}+B_{13} \lambda_{2}, \\
{ }_{4} \lambda_{2}=A_{03} \lambda_{2}+A_{13} \lambda_{1}+B_{13} \lambda_{3}, \\
{ }_{4} \lambda_{3}=A_{03} \lambda_{3}+A_{13} \lambda_{2}, \quad{ }_{4} \lambda_{4}=A_{13} \lambda_{3}, \quad{ }_{4} \lambda_{-1}=A_{03} \lambda_{-1}+A_{13} \lambda_{-2}+B_{13} \lambda_{0}, \\
{ }_{4} \lambda_{-2}=A_{03} \lambda_{-2}+A_{13} \lambda_{-3}+B_{13} \lambda_{-1}, \quad{ }_{4} \lambda_{-3}=A_{03} \lambda_{-3}+B_{13} \lambda_{-2}, \\
{ }_{4} \lambda_{-4}=B_{13} \lambda_{-3} .
\end{array}\right.
$$

At the rest of this section we will discuss and solve our problem for some particular cases for the Riccati equation as follows:

A. $c_{0}=c_{1}=1, c_{2}=0$. For this choice of the constants, the Riccati equation has the solution:

$$
\Lambda_{1}(\xi)=\exp (\xi)-1
$$

By the aid of Mathematica, the above system of equations (2.9) can be solved for the following cases:

Case 1. $A_{0}=\frac{3 \Psi}{16 \Theta}, A_{1}=0, B_{1}= \pm \sqrt{\frac{3\left(s l-k^{2} \omega\right)}{4 \Theta}}$. According to $(2.7),(2.8)$ and (2.10), Eq.(2.6) has the solution

$$
\chi_{1}(t, x, z)=\frac{3 \psi}{16 \theta} \pm \sqrt{\frac{3\left(s l-k^{2} \omega\right)}{4 \theta(\exp (\xi)-1)^{2}}}
$$

where 


$$
\begin{aligned}
& \xi=k x+k^{2} \int_{0}^{t} \omega(\tau, z) d \tau+\int_{0}^{t} \frac{4 \theta(\tau, z)}{18}\left\{\left(\frac{3 \psi(\tau, z)}{16 \theta(\tau, z)}\right)^{2} \psi(\tau, z)\right. \\
&\left.-\left(\frac{3 \psi(\tau, z)}{16 \theta(\tau, z)}\right) \phi(\tau, z)-\left(\frac{3 \psi(\tau, z)}{16 \theta(\tau, z)}\right)^{3}\right\} d \tau
\end{aligned}
$$

B. $c_{0}=-c_{2}=0.5, c_{1}=0$. For this choice of the constants, the Riccati equation has the solution:

$$
\Lambda_{2}(\xi)=\tanh (\xi) \pm i \operatorname{sech}(\xi)
$$

or

$$
\Lambda_{3}(\xi)=\operatorname{coth}(\xi) \pm \operatorname{csch}(\xi)
$$

By the aid of Mathematica, the above system of equations (2.9) can be solved for the following case:

Case 2. $A_{0}=\frac{3 \psi}{16 \theta}, A_{1}=\mp i \sqrt{\frac{3\left(s l-k^{2} \omega\right)}{16 \theta}}, B_{1}= \pm \sqrt{\frac{3\left(s l-k^{2} \omega\right)}{16 \theta}}$. According to (2.7),(2.8) and (2.10), Eq.(2.6) has the solution

$$
\begin{aligned}
& \chi_{j}(t, x, z)=\frac{3 \psi}{16 \theta} \mp i \sqrt{\frac{3\left(s l-k^{2} \omega\right)}{16 \theta}} \Lambda_{j}(\xi) \pm \sqrt{\frac{3\left(s l-k^{2} \omega\right)}{16 \theta}} \Lambda_{j}^{-1}(\xi), \\
& j=2,3 .
\end{aligned}
$$

where

$$
\xi=k x+k^{2} \int_{0}^{t} \omega(\tau, z) d \tau
$$

C. $c_{2}=4 c_{0}=1, c_{1}=0$. For this choice of the constants, the Riccati equation has the solution:

$$
\Lambda_{4}(\xi)=0.5 \tan (2 \xi)
$$

or

$$
\Lambda_{5}(\xi)=0.5 \cot (2 \xi)
$$

By the aid of Mathematica, the above system of equations (2.9) can be solved for the following case:

Case 3. $A_{0}=\frac{3 \psi}{16 \theta}, A_{1}=\mp i \sqrt{\frac{3\left(s l-k^{2} \omega\right)}{16 \theta}}, B_{1}= \pm \sqrt{\frac{3\left(s l-k^{2} \omega\right)}{16 \theta}}$. According to (2.7),(2.8) and (2.10), Eq.(2.6) has the solution

$$
\chi_{4}(t, x, z)=\frac{3 \psi}{16 \theta} \mp i \sqrt{\frac{3\left(s l-k^{2} \omega\right)}{16 \theta}} \tan (2 \xi) \pm \sqrt{\frac{3\left(s l-k^{2} \omega\right)}{16 \theta}} \tan ^{-1}(2 \xi) .
$$


and

$$
\chi_{4}(t, x, z)=\frac{3 \Psi}{16 \Theta} \mp i \sqrt{\frac{3\left(s l-k^{2} \omega\right)}{16 \Theta}} \cot (2 \xi) \pm \sqrt{\frac{3\left(s l-k^{2} \omega\right)}{16 \Theta}} \cot ^{-1}(2 \xi) .
$$

where

$$
\xi=k x+\frac{5 k^{2}}{6} \int_{0}^{t} \omega(\tau, z) d \tau
$$

At the end of this section we should remark that, there exists infinitely number of solutions for Eqn.(2.6) these solution coming from solving the system (2.9) with regarding the Riccati equation (2.8). The above mentioned cases are just to clarify how far my technique is applicable.

\section{White Noise Functional Solutions}

The main aim of the rest of this paper is to obtain white noise functional solutions of Eqs.(1.2). As pointed out from Xie [14], we will use Theorem 2.1 of for $d=2$. The properties of hyperbolic functions yield that there exists a bounded open set $\mathbf{S} \subset \mathbb{R}_{+} \times \mathbb{R}^{2}, m>0$ and $n>0$ such that $u(x, y, t, z), u_{x t}(x, y, t, z)$ are uniformally bounded for all $(t, x, y, z) \in \mathbf{S} \times \mathbb{K}_{m}(n)$, continuous with respect to $(t, x, y) \in \mathbf{S}$ for all $z \in \mathbb{K}_{m}(n)$ and analytic with respect to $z \in \mathbb{K}_{m}(n)$ for all $(t, x, y) \in \mathbf{S}$. Using Theorem 2.1 of Xie [14], there exists a stochastic process $U(t, x, y)$ such that the Hermite transformation of $U(t, x, y)$ is $u(t, x, y, z)$ for all $\mathbf{S} \times \mathbb{K}_{m}(n)$, and $U(t, x, y)$ is the solution of (1.2). This implies that $U(t, x, y)$ is the inverse Hermite transformation of $u(t, x, y, z)$. Hence, for $\Phi(t) \Psi(t) \Theta(t) \neq 0$ the white noise functional solutions of Eqs.(1.2) can be written as follows:

$$
U_{1}(t, x)=\sqrt{\frac{3 \Psi(t)}{16 \Theta(t)} \pm \sqrt{\frac{3\left(s l-k^{2} \Omega(t)\right)}{4 \Theta(t)\left(\exp ^{\diamond}(\Xi)-1\right)^{2}}}} .
$$

where

$$
\begin{aligned}
\Xi(t, x)=k x+k^{2} \int_{0}^{t} \Omega(\tau) d \tau+\int_{0}^{t} \frac{4 \Theta(\tau)}{18} & \left\{\left(\frac{3 \Psi(\tau)}{16 \Theta(\tau)}\right)^{2} \Psi(\tau)\right. \\
- & \left.\left(\frac{3 \Psi(\tau)}{16 \Theta(\tau)}\right) \Phi(\tau, z)-\left(\frac{3 \Psi(\tau)}{16 \Theta(\tau)}\right)^{3}\right\} d \tau
\end{aligned}
$$

and 


$$
\begin{aligned}
& U_{j}(t, x)=\sqrt{\frac{3 \Psi(t)}{16 \Theta(t)} \mp i \sqrt{\frac{3\left(s l-k^{2} \Omega(t)\right)}{16 \Theta(t)}} \Lambda_{j}^{\diamond}(\Xi) \pm \sqrt{\frac{3\left(s l-k^{2} \Omega(t)\right)}{16 \Theta(t)}} \Lambda_{j}^{-\diamond}(\Xi)}, \\
& j=2,3 .
\end{aligned}
$$

where

$$
\Xi(t, x)=k x+k^{2} \int_{0}^{t} \Omega(\tau) d \tau
$$

and

$$
\begin{aligned}
& U_{4}(t, x)= \\
& \diamond \sqrt{\frac{3 \Psi(t)}{16 \Theta(t)} \mp i \sqrt{\frac{3\left(s l-k^{2} \Omega(t)\right)}{16 \Theta(t)}} \tan ^{\diamond}(2 \Xi) \pm \sqrt{\frac{3\left(s l-k^{2} \Omega(t)\right)}{16 \Theta(t)}} \tan ^{-\diamond}(2 \Xi)}
\end{aligned}
$$

and

$$
\begin{aligned}
& U_{5}(t, x)= \\
& \diamond \sqrt{\frac{3 \Psi(t)}{16 \Theta(t)} \mp i \sqrt{\frac{3\left(s l-k^{2} \Omega(t)\right)}{16 \Theta(t)}} \cot ^{\diamond}(2 \Xi) \pm \sqrt{\frac{3\left(s l-k^{2} \Omega(t)\right)}{16 \Theta(t)}} \cot ^{-\diamond}(2 \Xi)}
\end{aligned}
$$

where

$$
\Xi(t, x)=k x+\frac{5 k^{2}}{6} \int_{0}^{t} \Omega(\tau) d \tau .
$$

\section{Discussions}

Our first interest in present work being in implementing the extended tanhcoth method, Hermite transform and white noise analysis to stress its power in handling nonlinear equations so that one can apply it to models of various types of nonlinearity. The next interest is in the determination of exact travelling wave solutions for modified KG equations. Also, we have presented Riccati equation expansion method and applied it to the modified KG equations. As a result, some new exact travelling wave solutions of the modified KG equation are obtained because of more special solutions of Eq.(2.1). The method 
which we have proposed in this letter is standard, direct and computerized method, which allow us to do complicated and tedious algebraic calculation. It is shown that the algorithm can be also applied to other NLPDEs in mathematical physics such as KdV-Burgers, Modified KdV-Burgers, Zhiber-Shabat equations (specially: Liouville equation, Sinh-Gordon equation, Dodd-BulloughMikhailov equation, Dodd-Bullough-Mikhailov equation and Tzitzeica-DoddBullough equation) and Benjamin-Bona-Mahony equations. Also, we remark that, since the Riccati equation has other solution if select other values of $c_{0}, c_{1}$ and $c_{2}$, there are many other exact solutions of variable coefficient and wick-type stochastic modified KG equation.

\section{References}

[1] M. Wazwaz, The tanh and the sine-cosine methods for compact and noncompact solutions of the nonlinear Klein-Gordon equation, Appl. Math. Comput., 167 (2005), 1196-1210.

[2] A.M. Wazwaz, Exact solutions of the generalized sine-Gordon and the generalized sinh-Gordon equations, Chaos, Solitons $\& 3$ Fractals, 28 (2006), 127-135.

[3] M. Wadati, Stochastic Korteweg de Vries equation, J. Phys. Soc. Jpn., 52 (1983), 2642-2648.

[4] M. Wadati, Y. Akutsu, Stochastic Korteweg de Vries equation, J. Phys. Soc. Jpn., 53 (1984), 3342-50.

[5] H. Holden, B. Øsendal, J. Ubøe and T. Zhang, Stochastic Partial Differential Equations, Birhkäuser, Basel, 1996.

[6] A. de Bouard and A. Debussche, On the stochastic Korteweg-de Vries equation, J. Funct. Anal., 154 (1998), 215-251.

[7] A. de Bouard and A. Debussche, White Noise Driven Korteweg-de Vries Equation, J. Funct. Anal., 169 (1999), 532-558.

[8] A. Debussche and J. Printems, Numerical simulation of the stochastic Korteweg-de Vries equation, Physica D : Nonlinear Phenomena, 134 (1999), 200-226.

[9] A. Debussche and J. Printems, Effect of a localized random forcing term on the Korteweg-de Vries equation, Comput. Anal. Appl., 3 (2001), 183-206. 
[10] H.A. Ghany, Exact solutions for stochastic generalized Hirota-Satsuma coupled KdV equations, J. Chin. Phys., 49 (2011), 926-940.

[11] H.A. Ghany, A. Hyder, Exact solutions for the Wick-type stochastic timefractional KdV equations, Kuwait Journal of Science, 41 (2014), 1-14.

[12] H.A. Ghany, A. Hyder, Abundant solutions of the Wick-type stochastic fractional 2D KdV equations, Chin. Phys. B., 23 (2014), 060503:1-7.

[13] H.A. Ghany, S. Mohammed, White noise functional solutions for the Wick-type stochastic fractional KdV-Burgers-Kuramoto equations, Chin. J. Phys., 50 (2012), 619-627.

[14] Y. Xie, Exact solutions for stochastic KdV equations, Phy. Lett. A, 310 (2003), 161-167. 